\title{
Improved Convolutional Neural Network Based Cooperative Spectrum Sensing For Cognitive Radio
}

\author{
Appala Raju Uppala ${ }^{*}$, Venkata Narasimhulu $C^{2}$, and Satya Prasad K \\ ${ }^{1}$ Research Scholar, Department of ECE, Jawaharlal Nehru Technological University \\ Kakinada Andhra Pradesh, India \\ Associate Professor, Geethanjali College of Engineering and Technology, \\ Hyderabad, Telangana, India \\ [e-mail: rekharajuppala2009@gmail.com] \\ ${ }^{2}$ LORDS Institute of Engineering and Technology, Hyderabad, \\ Telangana, India \\ [e-mail: narasimhulucv@gmail.com] \\ ${ }^{3}$ Vignan's Foundation for Science, Technology \& Research \\ Guntur, India \\ [e-mail: prasad_kodati@yahoo.co.in] \\ ${ }^{*}$ Corresponding author: Appala Raju Uppala
}

Received October 24, 2020; revised January 29, 2021; accepted May 16, 2021;

published June 30, 2021

\begin{abstract}
Cognitive radio systems are being implemented recently to tackle spectrum underutilization problems and aid efficient data traffic. Spectrum sensing is the crucial step in cognitive applications in which cognitive user detects the presence of primary user (PU) in a particular channel thereby switching to another channel for continuous transmission. In cognitive radio systems, the capacity to precisely identify the primary user's signal is essential to secondary user so as to use idle licensed spectrum. Based on the inherent capability, a new spectrum sensing technique is proposed in this paper to identify all types of primary user signals in a cognitive radio condition. Hence, a spectrum sensing algorithm using improved convolutional neural network and long short-term memory (CNN-LSTM) is presented. The principle used in our approach is simulated annealing that discovers reasonable number of neurons for each layer of a completely associated deep neural network to tackle the streamlining issue. The probability of detection is considered as the determining parameter to find the efficiency of the proposed algorithm. Experiments are carried under different signal to noise ratio to indicate better performance of the proposed algorithm. The PU signal will have an associated modulation format and hence identifying the presence of a modulation format itself establishes the presence of PU signal.
\end{abstract}

Keywords: Cognitive radio, Cooperative spectrum sensing, Primary user, Simulated annealing, Neural network. 


\section{Introduction}

In cognitive radio (CR) networks, the spectrum resources have become highly scarce [14] with the rapid advancement of 5G paradigm in advent of wireless connectivity technology [34]. Spectrum allocation will limit and avoid the interference between cognitive radio devices and licensed primary users to authorize a better methodical usage of the wireless radio spectrum [43]. The interface between spectrum availability and its development has been emerged as a potential growth in cognitive radio. Efficient granting of unused spectrum to unlicensed users is an important problem [28]. The identified licensed users are called primary users (PUs) [31] and unlicensed users are called secondary users (SUs) [25]. The main aim of spectrum sensing is to detect the spectrum holes of enormous number of primary users in the network [34]. A new spectrum sensing method which can distinguish all types of primary users based on the inherent ability has been proposed in this paper. It utilizes improved convolutional neural network with simulated annealing (SA) to discover the quantity of neurons in each hidden layer of the primary users. In addition, we discover the LSTM spectrum sensing that combines CR networks with CNN. The deep learning methods solve the optimization problem of forecasting the number of neurons. The proposed algorithm utilizes SA to discover reasonable number of neurons with low unpredictability in each layer of the completely associated CNN.

\subsection{Current State of the Art and Motivation}

Spectrum sensing is categorized into two types namely individual spectrum sensing and cooperative spectrum sensing. In individual spectrum sensing, only one secondary user can sense to know how many spectrum holes are available at a particular time. But in cooperative spectrum sensing, the secondary users can sense a bunch of available spectrum holes thereby improving the execution [33]. Energy detection is an important method used in spectrum sensing because of its less computational and execution complexities even with drawbacks in finding out the noise and the signal. Energy detection can be done by means of energy comparison between the received signals in specific frequency bands for setting a proper decision threshold [20]. Therefore, the output gives prior knowledge of the PU in this method of detection and causes signal to noise ratio (SNR) [26]. Non-parametric blind spectrum sensing like Censored Anderson Darling (CAD) [9], likelihood ratio statistics (LRS-G2G2) [30] and Anderson Darling test [41] is proposed in the literature. The occupancy patterns in PU activities consists of a number of factors like time, location and frequency band [3]. The individual SUs characteristic changes the channel condition of the spectrum. We used cooperative spectrum sensing (CSS) to predict the error and to conquer this issue. The results from numerous individual spectrum sensing data of secondary users are used for finding the availability of PU. Cooperative spectrum sensing (CSS) is used to overcome the channel impacts, to exploit location diversity and to recognize a weak primary signal [10].

Nowadays deep learning has become one of the popular algorithms compared to other conventional methods due to its performance gain [18][36]. In a deep neural network (DNN), the near ideal strategy is to change over the information of the contemplated measure of sample data by back-propagation algorithm. The major benefits of CR are cognitive ability that denotes the learning nature of the radio networks. This method is same as the machine learning (ML) system. Hence, ML systems are applied to CR networks [7][2][4]. Besides CNN [24], the orthogonal frequency division multiplexing (OFDM) based CR receiver detects 
the OFDM signal of the primary users. Hence, cognitive radio receiver is outfitted with different antenna-based energy detectors [19]. For DNN-based detection structure, the DNN-based likelihood probability proportion test (DNN-LRT) is proposed in [24].

Several spectrum sensing algorithms are presented by numerous researchers all over the world. For instance, CNN based cooperative spectrum sensing is used on wireless data. The authors addressed a deep cooperative sensing with CSS method for convolutional neural network in [22]. Authors in [23] have proposed a covariance matrix-aware CNN (CM-CNN)-based spectrum sensing algorithm. Additionally, the creators in [45] have proposed the fire-feature reuse detection convolutional neural network. The above-mentioned methods have good accuracy and problem solving skills when compared to other machine learning algorithms. On the other hand, it has its own limitations in predicting the available spectrum. To get rid of this issue, long short-term memory (LSTM) design has implemented a traditional method of recurrent neural network (RNN) for time and arrangement issues [23]. LSTM comprises some gates which have a neuron that readily arranges its verifiable data and the traffic type data [11] can be resolved precisely with LSTM. Moreover, LSTM based spectrum sensing (LSTM-SS) with CNN is proposed for CR networks. Further, CR users obtain beneficiary advantages from the PU in spectrum sensing decisions. Finally, we can find out the presence of PU by using this method.

An improved method has been introduced in deep learning methods. Yet, there is an issue with this neural network that the computational cost is very high [17]. For example, to shift through the noise we utilized advancement of an autoencoder to pack data from the info layer. To improve the results, we additionally found the hereditary algorithm which predicts an enlarged structure for deep learning scheme. In order to develop a powerful detecting approach, the proposed calculation receives deep neural system and metaheuristic calculations called simulated annealing for deep neural network (SADNN).

\subsection{Contributions}

The contribution of this paper is discussed below.

(1) First, the proposed method introduces deep learning-based spectrum sensing indicator to utilize CNN to exploit the energy connection features from the covariance framework of detecting information. Then, the arrangement of energy correlation features the comparison between various detecting periods of the LSTM. Hence, the action patterns of PUs can be learned.

(2) Secondly, the proposed strategy could investigate the discriminative features to additionally improve the detection performance. The LSTM structure is used to exploit the PU activity pattern. The proposed CNN-LSTM structure is free from signal-noise model assumptions. To make LSTM with fair high SNR, the preparation data set is set-up to incorporate the data at exceptionally low SNRs in varying values.

(3) Finally, the best boundaries of convolutional neural network are chosen by simulated annealing algorithm to improve the exactness of recognition and limit the computational multidimensional nature.

\subsection{Paper Organization}

The rest of this paper is structured as follows. Section 2 briefly depicts the background methodology. Section 3 describes the system model. CNN-LSTM is proposed in Section 4. Experimental outcomes are examined in Section 5 and in Section 6, conclusions are finally summarized. 


\section{Background Methodology}

The major problem in cognitive radio is raised due to the inadequacy of spectrum resources and mismanagement of spectrum [35]. Adapting techniques like cyclo-stationary detection (CD), matched filter detection (MFD), energy detection (ED) and ML techniques enhance the functioning of CSS in cognitive radio systems. Energy detection is one of the traditional schemes that recognize all forms of primary users inside the transmission range of a CR user. Especially, the OFDM based CR receiver recognizes the primary user OFDM signal where CR receiver is furnished by different antenna-based energy detectors [19]. The two basic types of spectrum sensing in cognitive radio setup are cyclo-stationary detection and energy detection. Both of these techniques detect the licensed users in cognitive radio setup. Analysis of these two techniques is carried out in [37] to bring an improved technique for primary user identification. Cooperative spectrum sensing in OFDM dependent on energy recognition in multiple-input multiple-output (MIMO) cognitive radio sensor networks is implemented. Soft combination of the observed energy esteems various cognitive radio users is examined. OFDM based MIMO cognitive radio network recognizes the OFDM signal with multiple antenna detector of the CR receiver. It resulted in the critical development of primary user identification of the square law combining with energy detection of a MIMO cognitive radio sensor networks [46].

Machine learning techniques are adopted in several works [32][13] to allow CR users to be trained about the nature of the system. This can be carried out by techniques that use both supervised and unsupervised learning approaches or only supervised learning approach. In the first type, algorithms like k means which uses PU transmission patterns and statistics such as energy statistic and probability vector are used. Then, the identification of primary user is done by training the classifier using labelled training data [40]. In the second case, the primary user can be recognized by using classifiers like K-nearest neighbour (KNN), support vector machine (SVM) or naïve bayes. The effectiveness of these machine learning algorithms is completely dependent on feature selection. In case of KNN classifier, spectrum sensing features are needed for $\mathrm{K}$ neighbour classes. A test case is then ordered into one of the $\mathrm{K}$ neighbours based on larger part casting a vote. The voting depends on the statistical data picked up for pointing out the separation between the test case and the training instances. The separation ought to be determined precisely and really reflects the classifying classes [16].

Artificial neural network (ANN) based spectrum sensing (SS) is advanced to evaluate the database and to prepare the ANN to distinguish between signal and noise [51]. In cognitive radio, spectrum sensing has been considered as an ideal method to sense the availability of primary users in the network. Here, ANN analysis is to detect the signals and hence performance error has been occurred [36]. Due to this limitation, cooperative spectrum sensing is proposed in the duty cycle detection model for primary users [29].

Deep learning algorithms for parallel CNN-LSTM spectrum sensing is proposed where the signal and the noise prepare the model to detect multiple signals. Different patterns were performed to reveal the accuracy of the proposed scheme that does not require earlier information of the licensed user [27]. At that point, deep cooperative sensing (DCS) includes the first CSS system dependent on a CNN. In DCS, the framework for combining the individual detecting consequences of the SUs is found out freely with a CNN utilizing prepared detection tests, whether or not the individual detection outcomes are quantized. Furthermore, spectral and spatial correlations of detecting the results are considered as CSS in DCS [22].

Currently, spectrum sensing addresses much on deep learning algorithm. CNN-LSTM detector uses CNN for energy correlation to extract the information. The sensing information 
of different periods is inserted as an input for LSTM to identify the primary user activity [49]. The blind spectrum sensing technique that depends on deep learning is composed of three types of neural systems like convolutional neural networks, long short-term memory and fully connected neural networks [15]. DNN-based likelihood probability proportion test is tested in DNN-based detection frameworks. DNN-based system is used as the input of a CNN to present a covariance matrix-aware CNN (CM-CNN)-based spectrum sensing algorithm [24].

The sensing performance in deep neural networks got increased but there are no strategies to analyse the variables of deep learning and to solve the optimization. Additionally, DNN is proposed to find out the quantity of neurons in the layer [20, 47 \& 8]. Systems like autoencoder can be used to resize the information (dimensionality decrease) data to limit the disadvantages [21]. An unsupervised learning algorithm named genetic algorithm (GA) is utilized in [48] to recognize the best structure of DNN. Simulated annealing based deep neural network is utilized to improve the precision rate and to build up a beneficial detecting strategy by picking the best limits.

In spite of the fact that countless spectrum detecting strategies are accessible in the literature, every method has restriction in any of the three fundamental CR necessities like low detection time, low multidimensional nature and precision of identification. Strategies like cyclostationary feature detection (CFD) and OFDM require earlier information on PU signal in which CR situation is not fitting. Majority of other spectrum detection methods are incredibly mind boggling with high inactivity which is unacceptable for mechanical sensor and actuator systems. Therefore, it is important to structure a detecting procedure which is anything but difficult to execute and identify all types of PU signals. Subsequently, this article has proposed another spectrum sensing procedure that can identify a wide range of PU signals (both solid and feeble, with or without earlier data) with low unpredictability and improved determinism. However, CNN-LSTM is free from signal to noise model presumptions. To make LSTM with fair high SNR, the preparation informational index is set-up to incorporate information at low SNR in changing extents. SA is utilized to discover an appropriate quantity of neurons for every layer of a completely associated DNN. It upgrades the correct value in taking care of the specific enhancement issue. Contrasted with efficiently listing technique, it can discover the rough arrangement inside a sensible time. The proposed novel spectrum detecting strategy is planned for identifying the PU with least time and improved exactness.

\section{System Model}

Cognitive radio network comprises of PUs and SUs. The SUs in the CR network consider the spectrum band to sharply get to the white space. Here, two metrics such as $H 0$ and $H 1$ were considered with respect to the condition of the PUs in Equation ( 1 \& 2). In this, $H 0$ represents the absence of PU and $H 1$ signifies the time of PU presence.

$$
\begin{gathered}
H 1: x(n)=s(n)+u(n) \\
H 0: x(n)=u(n)
\end{gathered}
$$

Where $x(n)$ is the $n$-th received signal, $s(n)$ indicates the signal from PU, $u(n)$ signifies the additive white Gaussian noise (AWGN) sample. The spectrum sensing works on a principle that an unlicensed secondary user can utilize the spectrum resources when a licensed user is inactive or absent. Thus, the exhibition of spectrum sensing is assessed by the probability of detection and the probability of false alarm. The detection probability is the probability of choosing $H 1$ when $H 1$ is valid; the probability of false alarm is the probability that the choice is $H 1$ when $H 0$ is valid. In cooperative spectrum sensing, the secondary user 
can sense a bunch of available spectrum holes to sense the execution. Hence, the statistical covariance can be calculated using a limited number of signal samples. For notation simplicity, we consider the case of single sensor with $\mathrm{M}=1$. Fig. 1 shows the system model of cooperative spectrum sensing.

\subsection{Pre-Processing}

To represent as a simple process, we illustrate the entire received signal samples in the u-th detecting time interval as in Equation (3).

$$
M_{i}=\left\{M_{i}(1), \ldots \ldots \ldots \ldots M_{i}(N)\right.
$$

In deep learning algorithms, labelled training data is needed where the training data of the proposed method is defined as given in Equation (4).

$$
M=\left\{\left(M_{1}, l_{1}\right),\left(M_{1}, l_{2}\right), \ldots \ldots \ldots\left(M_{n}, l_{n}\right)\right\}
$$

Where, $n$ is the size of the training set and $l_{n}$ is the label. The label is the actual PU activity state corresponding to $M_{n}$. The real primary user activity state corresponds to $M_{n}$ as the computation difficulty of the neural network is partially linked with the size of the input $M_{n}$. Furthermore, $M_{n}$ is created from monotonous sampling of comparative dispersion which has overabundant information. Subsequently, we pre-process the sensing samples before contributing into the neural network. The labelled training data can be obtained by cooperating with the PU. The fundamental fact of PU activity must be known.

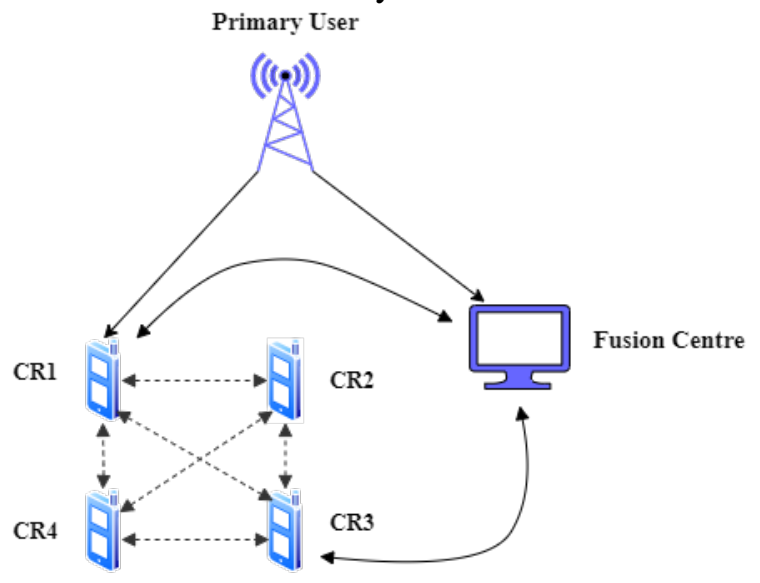

Fig. 1. System model of cooperative spectrum sensing

\subsection{Sample Covariance Matrix}

Sample covariance matrix is one of the adaptable statistics which inherently consolidates diverse discriminative i.e., energy, eigenvalues and non-diagonal elements. Hence, sample covariance matrix is proposed to act as an input to the neural network even though it does not have the energy correlation data of the sensing samples. Here, the sample covariance matrix is mentioned in Equation (5).

$$
P s=\frac{1}{Z} N_{m} N_{m}^{J}
$$

So, the pre-processed training dataset is expressed as in Equation (6).

$$
P s=\left\{\left(P_{1}, l_{1}\right),\left(P_{1}, l_{2}\right), \ldots \ldots \ldots \ldots\left(P_{m}, l_{m}\right)\right\}
$$


Where, $P_{m}$ denotes the sample covariance matric and $l_{m}$ indicates the corresponding label. $N_{m}$ represents all the received signal samples in the $\mathrm{m}^{\text {th }}$ sensing period. $m$ is the size of the training set, $Z$ is the signal sample based on the PU activity, $J$ is the channel index between PU and SU. Additionally, various sensing period of the spectrum sensing is processed to predict on-off strategy of the PU. In this paper, the proposed CNN extracts the energy correlation features from the covariance matrices created by the sensing data. At that point, the energy-correlation function contributed to various sensing periods are taken as input and processed for generalized neural networks to study the activities of the primary user.

\section{Spectrum Sensing by Improved Convolutional Neural Network}

\subsection{Proposed Method}

The improved neural network possesses the ability to remove the features and map the values. The three-layer structure such as input layer, hidden layer and output layer faces enormous number of issues in the variables. CNN is a multilayer structure where the characters are predicted by itself. Moreover, it is a feed forward network with structural parts of the neural network. The problem in this network is solved by using simulated annealing based hidden layer modelling. The extracted features from the convolution and pooling layer of CNN are passed to the LSTM layer. At last, CNN-LSTM model is used to predict the generated output of the network. The result and analysis of this proposed model can be demonstrated by few performance metrics.

\subsection{Dataset Construction}

Labelled training data is acquired by PU, while the PU activity is recognizable. To detect the execution process, we contemplate four digitally balanced families as the candidate set. Three different sets namely the training set, validation set and test set are considered. A wide range of SNR is sort out between $-20 \mathrm{~dB}$ and $18 \mathrm{~dB}$ with a break of $1 \mathrm{~dB}$ to deal with most of the executed situations. The AWGN information is created as noise information in the training set. Then, 100 samples are taken as training set for each SNR. The highlights of dataset factors are shown in Table 1.

Table 1. Dataset generation

\begin{tabular}{|c|c|}
\hline Modulation family & FSK, PAM, PSK, QAM \\
\hline \hline Signal length & 1024 \\
\hline Over sampling rate of signals & 2 \\
\hline Roll of factor & 0.35 \\
\hline No. of SNR levels & 20 \\
\hline Range of SNR & -20db to $18 \mathrm{db}$ \\
\hline Samples taken per modulation type & 8000 \\
\hline
\end{tabular}

In this method, 5000 samples are taken for each SNR that ranges between $20 \mathrm{db}$ and $18 \mathrm{db}$. There is similar number of PU signal and AWGN sequence. The generated information is split into training and testing datasets. Fig. 2 shows the dataset construction. 


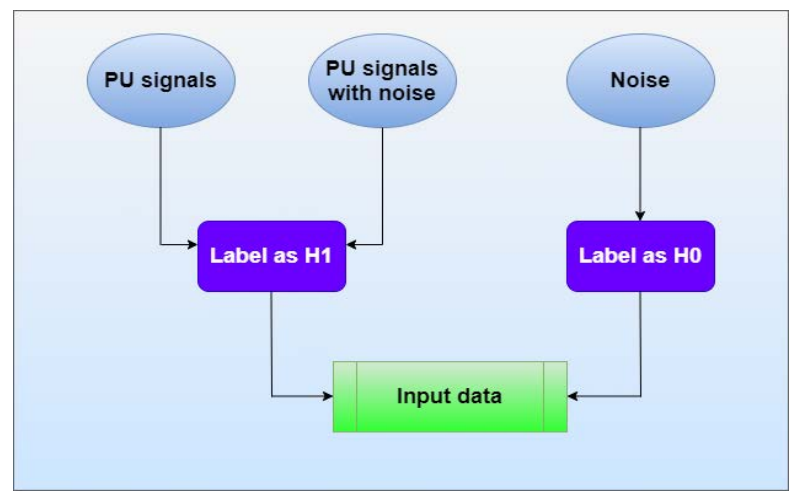

Fig. 2. Dataset construction

\subsection{Long Short-Term Memory (LSTM)}

Hochreiter et al. [11] proposed an LSTM as a specific variant of recurrent neural network. RNNs use the best gradient descent technique for learning about the research. However, it has its own limitation as gradient vanishing or detonation problem. To avoid these limitations, LSTM network has been introduced. The cell structure of LSTM unit is displayed in Fig. 3. It is comprised of three gates in particular input gate $i$, forget gate $f$ and output gate $o$. The computation procedure of LSTM unit at time $t$ is denoted.

$$
\begin{gathered}
I_{S}=\sigma\left(Y_{i} a_{s}+V_{i} d_{s-1}+g_{i}\right) \\
F_{S}=\sigma\left(Y_{f} a_{s}+V_{f} d_{s-1}+g_{f}\right) \\
O_{S}=\sigma\left(Y_{o} a_{s}+V d_{s-1}+g_{o}\right)
\end{gathered}
$$

Equations (7-9) explain the operation of input gate $i$, forget gate $f$ and output gate $o$ in which $I_{S}, F_{S}, O_{S}$ are the activation functions. $g_{f}$ is the offset term of forgetting gate.

$$
\begin{gathered}
C_{S}=F_{s} \bullet C_{s-1}+i_{t} \bullet \tanh \left(Y_{c} a_{t}+V_{c} d_{s-1}+g_{c}\right) \\
O_{S}=\sigma\left(Y_{o} a_{s}+V_{o} d_{s-1}+g_{o}\right)
\end{gathered}
$$

Equations 10 and 11 determine the cell state and the hidden state. The variable $a_{t}$ denotes the critical qualities of electric energy consumption and the output pooling layer at time $t . C_{S}$ is the hidden unit memory, $d_{s}$ is the corresponding unit output, $a_{s}$ is the unit input, $Y$ and $V$ are the weights, $g$ is bias variables, ' $\bullet$ ' indicates the Hadamard product and $\sigma \square$ means the activation function (tanh). LSTM algorithm is explained in Pseudocode 1.

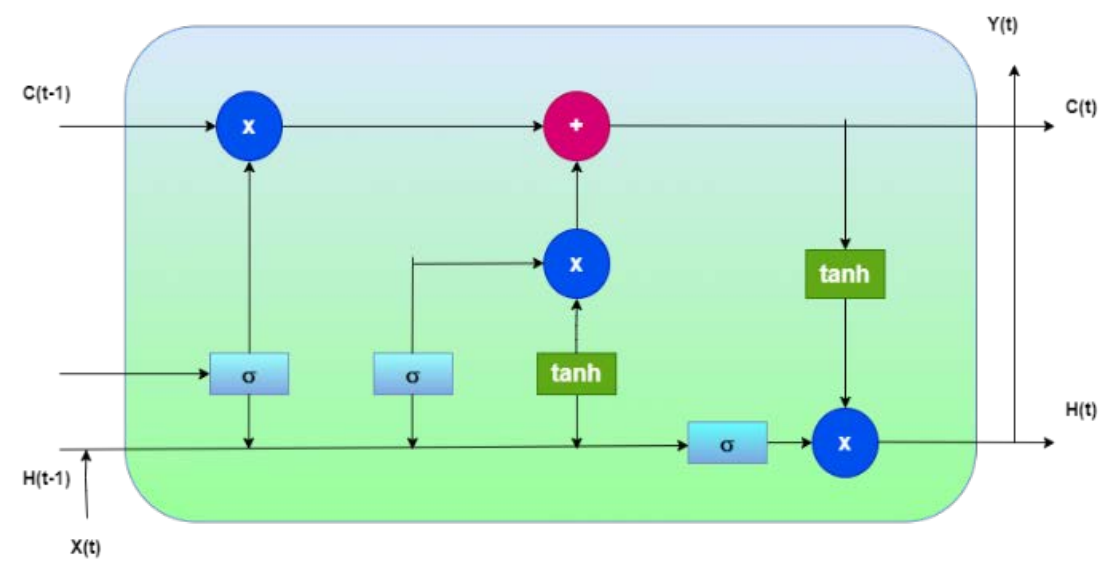

Fig. 3. LSTM structure 


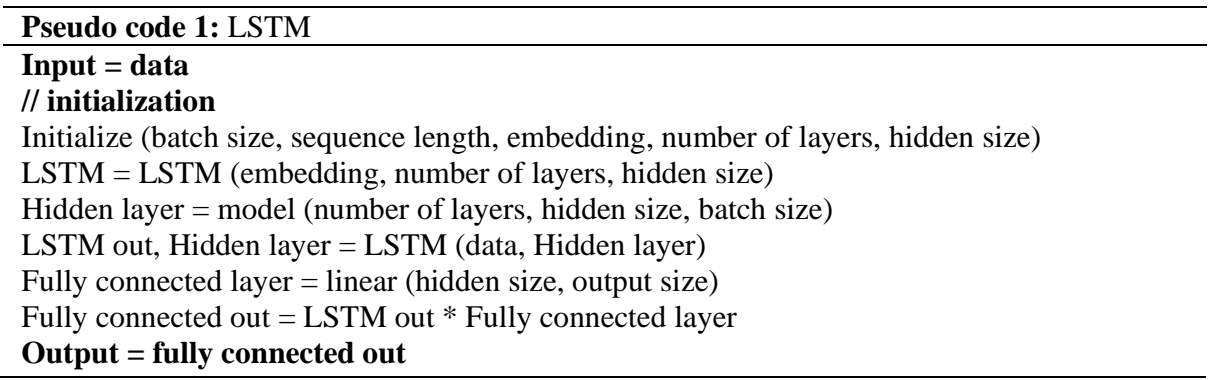

\subsection{Simulated Annealing for Convolutional Neural Network}

The main aim of SA is to find out the exact directions to delineate an assumed arrangement during the iterative process. When related to other methodologies, it is much better because of its accurate and low computational time. As seen in pseudocode 2, it comprises some associated factors: initialization, neighbour selection, evaluation of output, where t denotes the temperature. The neighbour selection operator of SA is assumed to change the search direction; evaluation operator estimates the number of solutions generated by neighbour selection. As appeared in lines 6-8 of Pseudocode 2, the search path of SA is related to probabilistic acceptance criterion. The temperature $t$ will be refreshed towards the end of every emphasis to diminish the diversity gradually with the goal of SA to few specific locales.

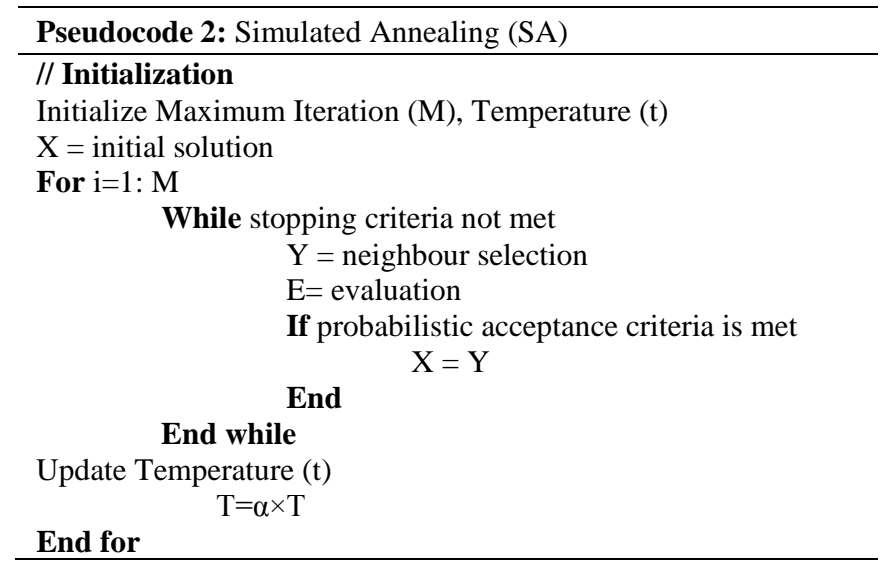

As shown in Pseudocode 3, the proposed dual algorithm integrates simulated annealing with CNN to form a unique search algorithm. The flow chart of SA-CNN is shown in Fig. 4. The input is considered as data $D$ and $t$ is another input parameter to determine the strategies of SA algorithm. Therefore, the proposed method is same as simple SA which plays the important role in determining the CNN solution. The initial role of SA is to predict the quantity of neurons in every hidden layer of the CNN. Hence, the identified outcome $Y$ of simulated annealing moves to the CNN structure and demonstrates certain illustration to examine the best model in the network. The proposed method will decide whether to keep the result $Y$ or not to determine the number of neurons. Then, as usual, the temperature $t$ will update the iterations at the end of the algorithm. The temperature gets updated by geometric reduction law as ( $T=\alpha \times T$, Where $\mathrm{T}$ is taken as $50^{\circ} \mathrm{C}$ and $\alpha$ reduction temperature is 0.95$)$. As temperature raises slowly, the neurons in the hidden layer gets affected. At certain temperature, the neurons have very low sound of spikes which finally gets discharged. In spite, SA will converge to some particular regions to improve the results for better structure of convolutional learning algorithm. The hidden layer in CNN gets affected by a metaheuristic algorithm (SA). 
SA finds the number of neurons in each layer of CNN to enhance the execution of CNN. Finally, the proposed CNN algorithm as shown is utilized to estimate the occurrence of primary user.

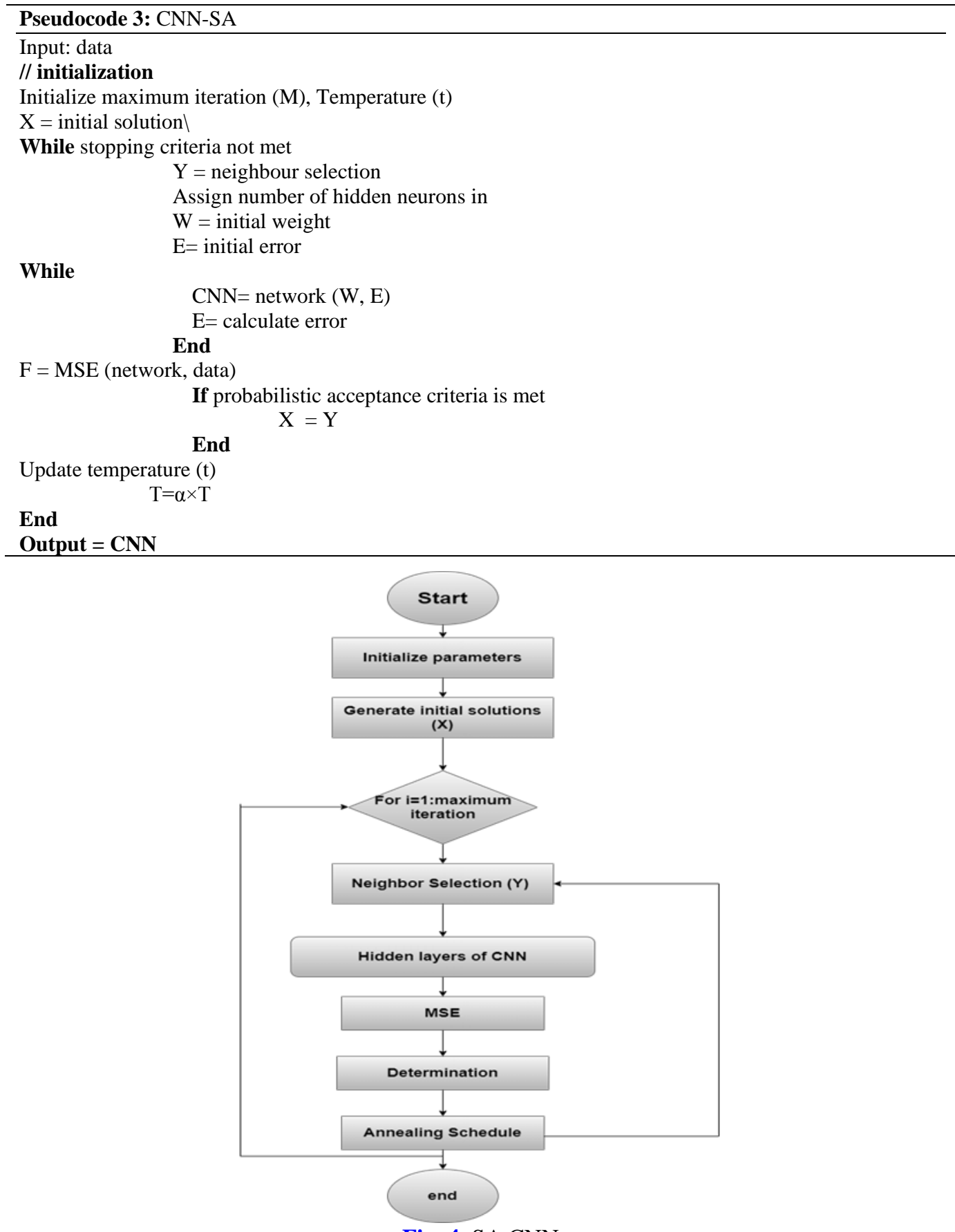

Fig. 4. SA CNN

\subsection{CNN LSTM Network Structure}

CNN-LSTM network structure is displayed in Fig. 5. The proposed CNN-LSTM network can obtain complex spatial and temporal features. CNN can extract features among several 
variables and LSTM layer can perform appropriate modelling with the temporal information. LSTM is the last layer of CNN-LSTM structure that stores important data extracted over CNN. LSTM provides a solution for preserving the long-term memory by consolidating memory units that can update the previous hidden state. This makes temporal features on long-term sequence.

The sample covariance matrices are complex which can be seen as two-layer process. The energy of the covariance matrix under state $\mathrm{H} 0$ is concentrated to the diagonal components in the real part while $\mathrm{H} 1$ energy is dispersed over the imaginary part and the real part. This visual distinction in covariance matrices under $\mathrm{H} 0$ and $\mathrm{H} 1$ makes the CNNs appropriate for studying the energy and correlation features out of the information. In CNN-LSTM module, sensing the data of CNN extracts features from the covariance matrices. The sequence of energy correlation features corresponding to numerous sensing periods are taken as the input of LSTM. The vectorized output consisting of energy and correlation features of the CNN sensing period are used as input to the LSTM cells. Likewise, the following strategy is to remove the time dynamic features in the array of sensing periods. Hence, the last output of LSTM cell is comprised of energy, correlation and time dynamic features of the sensing period is input into a thick layer to modify the output measurement as indicated by the quantity of information classes. Hence, PU activity pattern can be acquired.

\subsection{Network Training}

First introduce weights and biases in all convolution kernels. The forward propagation with the training set output $O$ is determined. At that point, the CNN can become familiar with the error $E$ through contrasting the output $O$ with the labels $y$. Assume the quantity of sample set as $S$ and the quantity of the sample types as $c$. We can compute the error $E$ as per the equation (12).

$$
E^{S}=\frac{1}{2} \sum_{m=1}^{S} \sum_{i=1}^{c}\left(y_{i}^{m}-O_{i}^{m}\right)^{2}
$$

CNN tests converges as stated by the value $E$. If it converges, then the training is finished. If it is not, then the residual $\delta$ of the output is analysed. Given the activation function $f$, the residual and the relu function is identified as activation function in the experiment. The residues can go from output layer to front layer. From Equation (13), residual can be calculated from each layer in which $\delta$ is the residual of the $\mathrm{l}^{\text {st }}$ layer.

$$
\delta=\left(\left(W^{l}\right)^{T} \delta^{(l+1)}\right) \cdot f^{\prime}\left(z^{l}\right)
$$
(15).

Update the weights and bias in each layer with the learning rate $\alpha$ as in Equations (14) and

$$
\begin{gathered}
W^{l}=W^{l}-\alpha \cdot \delta^{(l+1)}\left(a^{l}\right)^{T} \\
B_{i}^{l}=B_{i}^{l} \cdot \alpha \cdot \delta^{(l+1)}
\end{gathered}
$$

CNN continuously runs the above process to get an ideal outcome. It sends feature vector into NN for computing the training set. At any cost, the training set of a neural network is mathematically high. So, the performance is needed to be enhanced. To overcome this limitation, the introduced SA algorithm is used to find out the total quantity of neurons in the CNN. This solves the specific optimization problem and gives better accuracy rate. 


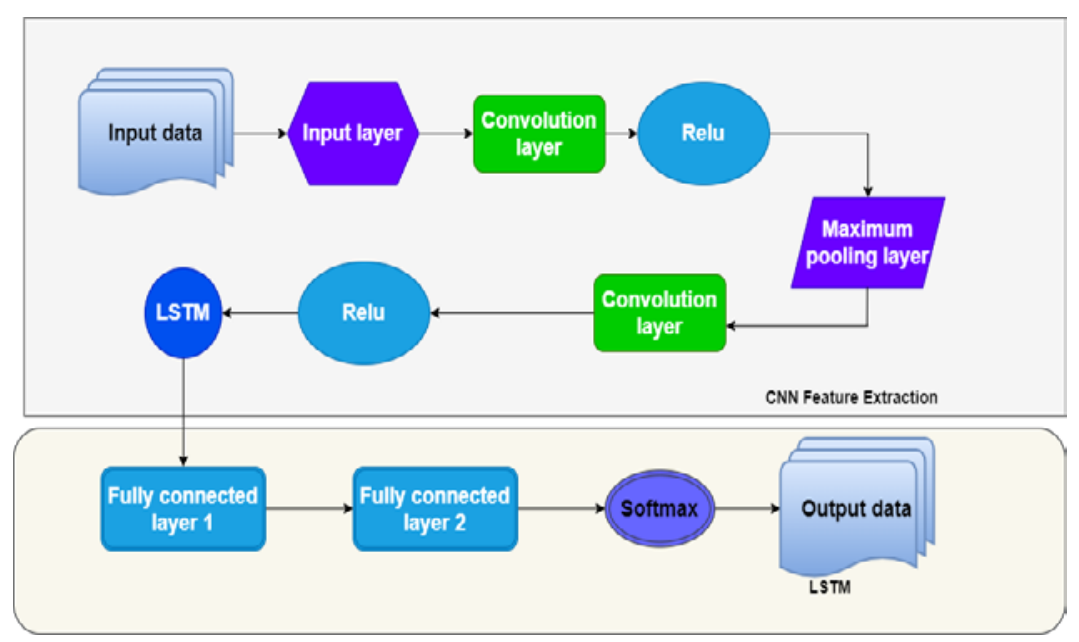

Fig. 5. CNN LSTM Structure

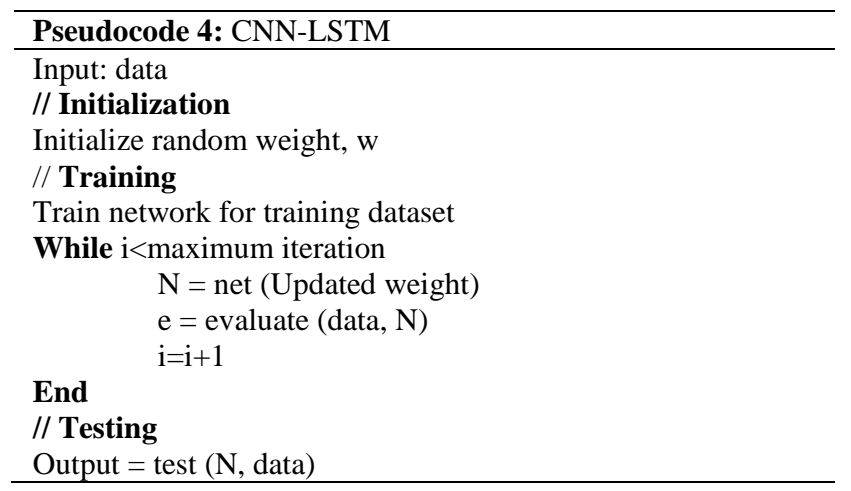

\section{Experimental Setup}

The proposed CNN-LSTM based spectrum sensing technique has been implemented in MATLAB R2018a. The datasets used in the proposed technique consist of SNR value in the range of $-20 \mathrm{db}$ to $18 \mathrm{db}$. The dataset is created with enormous samples with low SNR value. The aim of the proposed method is to point out the occupancy of PU signal even in low SNR value. Hence, $80 \%$ samples are maintained with low SNR value and $20 \%$ samples with high SNR range. Experiment is carried out by differentiating the number of training samples. Finally, a total number of 5000 samples are maintained for training process. The dataset composition is presented in Table 2.

Table 2. Composition of dataset

\begin{tabular}{|c|c|c|c|}
\hline Dataset & Label & Samples & Total \\
\hline \hline \multirow{2}{*}{ Training } & H0 & 2500 & \multirow{2}{*}{5000} \\
\cline { 2 - 3 } & H1 & 2500 & \\
\hline \multirow{2}{*}{ Testing } & H0 & 250 & \multirow{2}{*}{500} \\
\cline { 2 - 3 } & H1 & 250 & \multirow{2}{*}{5} \\
\hline
\end{tabular}

Specific parameters and the network architecture are chosen for the best performance of detector. The parameter setting used in the proposed CNN LSTM structure and the network architecture is presented in Table 3 and Table 4. 
Table 3. Composition of dataset

\begin{tabular}{|c|c|}
\hline Parameter & value \\
\hline Learning rate & 0.0005 \\
\hline Dropout rate & 0.1 \\
\hline Mini Batch size & 25 \\
\hline Number of epochs & 200 \\
\hline Number of hidden layers & 6 \\
\hline Activation function & Relu \\
\hline Loss function & Mean Squared Error (MSE) \\
\hline Training set & 5000 \\
\hline Testing set & 500 \\
\hline
\end{tabular}

Table 4. Network architecture

\begin{tabular}{|c|c|}
\hline Activation & Relu \\
\hline Optimizer & Adam \\
\hline Convolutional layer 1+relu & 250 \\
\hline Maximum pooling & $2 \times 2$ \\
\hline Convolutional layer 2+relu & 500 \\
\hline LSTM nodes & 124 \\
\hline Fully connected layer 1 & 62 \\
\hline Fully connected layer 2 & 2 \\
\hline
\end{tabular}

\subsection{Performance Evaluation}

Here, we assess the proposed CNN-LSTM spectrum sensing for its demonstration. The detection probability $(\mathrm{Pd})$ and the false alarm probability (Pf) are validated for assessing the proposed strategy. Signal information about the test dataset is taken individually from the system to determine Pd and Pf. It accurately arranges the signal, for example, (H1) is separated by the absolute number of PU signal models to decide Pd. In LSTM network, the AWGN sequence samples are inserted and the Pf is obtained. At any point, it could not predict HO where it was separated by AWGN sequence samples. The demonstration of the invented CNN LSTM structure for changing SNR ranges is introduced in Fig. 6 - 10. From the given experimental analysis, it is demonstrated that the proposed spectrum sensing strategy beats the current approaches.

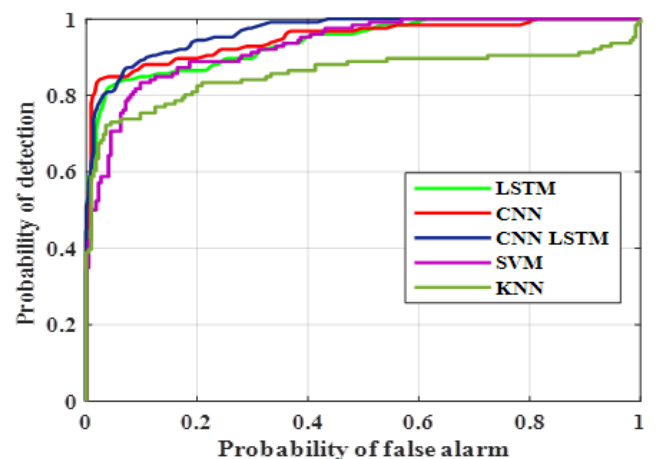

(a)

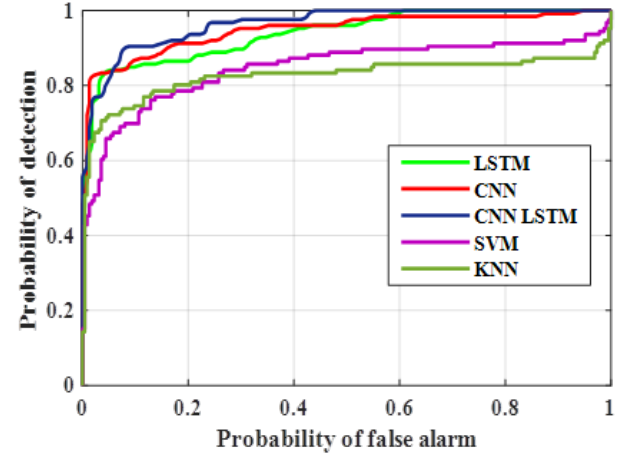

(b)

Fig. 6. Probability of detection (Pd) Vs Probability of false alarm (Pf) (a) SNR=-12db (b) SNR= -14db 
Fig. 6(a) shows the analysis of Pd (vs.) Pf under a dataset with SNR=-12db. It illustrates that the proposed CNN-LSTM method beats other sensing methods with less SNR. This is achieved due to the temporal characteristics available in the signal of the proposed CNN-LSTM method. The scientific reason behind the proposed CNN-LSTM method is their standard architecture of the network. Even though ANN has a shallow neural network, the LSTM has chain network. SVM considers the spatial temporal detector. The proposed method is the combination of deep neural network and looping structures. The elements are unrolled in a continuous manner in which other techniques do not perform this function.

The signal to noise ratio is set as $-14 \mathrm{db}$ in Fig. 6(b) to reveal the features of the proposed CNN-LSTM detector under different values. It represents that the CNN LSTM method has high density value when compared to other techniques like CNN and LSTM. The high false alarm probability in the existing KNN and SVM means that there is a less availability of primary users to use the spectrum holes. The proposed method contains various discriminative features that have the ability to extract features of matrix shaped data. CNN itself has the quality of relieving graphical features but it is not good at specifying the temporal features. Therefore, the proposed method of CNN-LSTM is good at sensing the temporal characteristics in the signal where other methods are unable to do so.

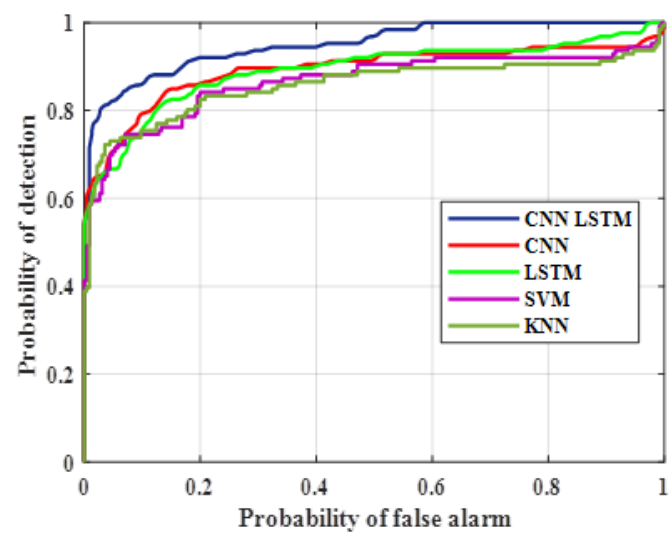

(a)

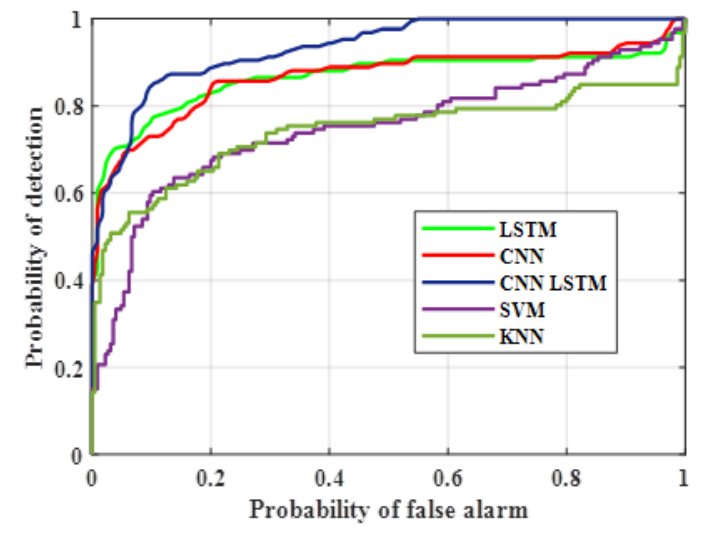

(b)

Fig. 7. Probability of detection (Pd) vs. Probability of false alarm (Pf) (a) SNR = -16db (b) SNR =-18db

The data is kept same for all three methods with SNR of -16db as shown in Fig. 7(a) to demonstrate a reasonable comparison with the proposed CNN LSTM method and other techniques. This is further used to verify whether Pd (vs.) Pf has high and accurate detection value. The major reason that the proposed CNN-LSTM method overcomes similar existing strategies as LSTM and CNN is due to the design and working principle of CNN-LSTM method. The deep feature extraction and fair detection capability of the proposed method extracts all features of the signal. From the plot, we can derive that the proposed CNN-LSTM method has high detection value than the other methods at a low SNR of -16 db. The proposed method has high probability of detection value than the machine learning algorithm namely KNN and SVM. Note that in Fig. 7(b), it shows the test statistics of the CNN LSTM technique by using the training set containing SNR value of -18db. It is shown that the proposed method has high detection value when compared to other proposed method like CNN, LSTM, SVM and KNN. The high performance detection is due to the CNN-LSTM structure which is free from signal to noise model. It simultaneously performs both energy correlation and temporal 
primary user detection. Though, CNN only performs to learn spatial features and LSTM performs only temporal features. The sensing efficiency of KNN and SVM classification speed is minimal. Hence, the invented method has best Pd (vs.) Pf even in the SNR value which is shown in Fig. 7(b).

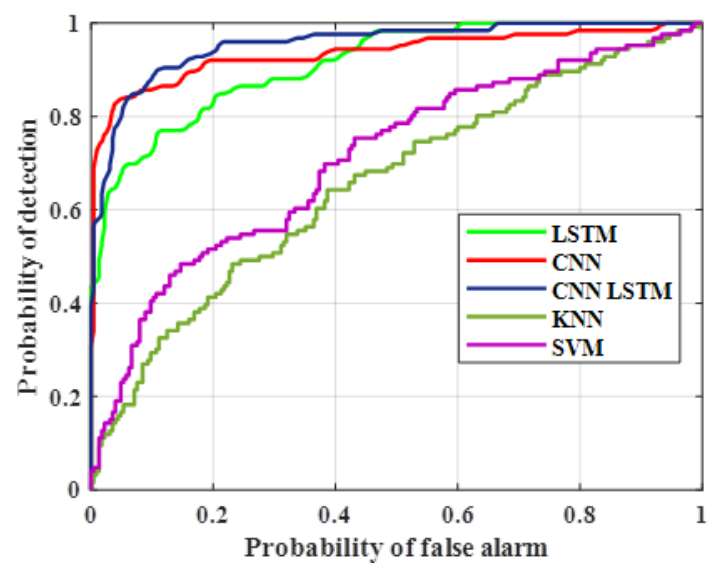

Fig. 8. Probability of detection (Pd) vs. Probability of false alarm (Pf) (SNR=-20db)

To execute the performance of the proposed CNN-LSTM with the existing methods, we evaluate the graph which shows Pd (vs.) Pf in Fig. 7 with the SNR value of $-20 \mathrm{db}$. This different SNR performance shows the best accuracy schemes of CNN-LSTM method. The data flow in LSTM is structured by the Gate layer as it performs extremely in temporal data. The CNN structure evaluates the energy correlation features where CNN-LSTM took all the hidden characteristics from both the invented information which definitely LSTM and CNN neglected to do as such. As well as the supervised machine learning techniques KNN, SVM algorithm required more time for training the classifier.

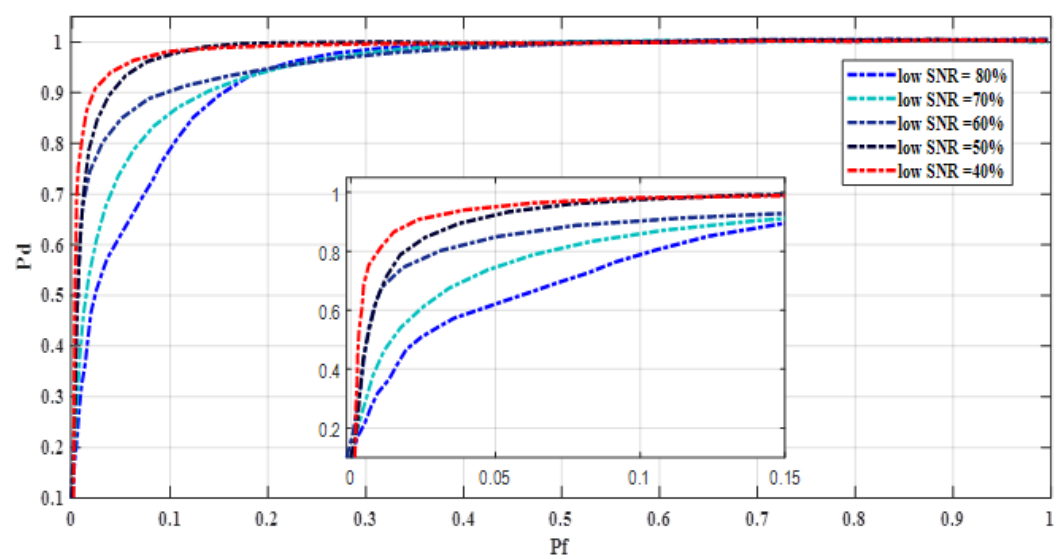

Fig. 9. Pd Vs Pf for various training samples

The creation of training set notably affects Pd and Pf from Fig. 9. For different SNR training sets, the Pf and Pd got enlarged. As the quantity of samples and level of samples in low SNR go is expanded, Pf and Pd likewise increment. The magnitude of the primary user signal is noted to be noise at low SNR. Network, in this manner, consider that it is difficult to separate the primary user signal and noise. This is exploited because the temporal correlation is placed in this method so that the plotted graph shows high detection value for the low SNR range at various samples for the proposed CNN LSTM work. 
The detection probability of various techniques for different SNR values is shown in Fig. 10. The various SNR values are compared with detection probability of different techniques like CNN, LSTM, SVM and KNN. When the SNR values get reduced, the detection probability also gets decreased. The proposed spectrum sensing method provides high probability of detection when compared with other techniques. This seems that the CNN-LSTM has good temporal dependency and it outperforms other schemes as shown in the bar diagram.

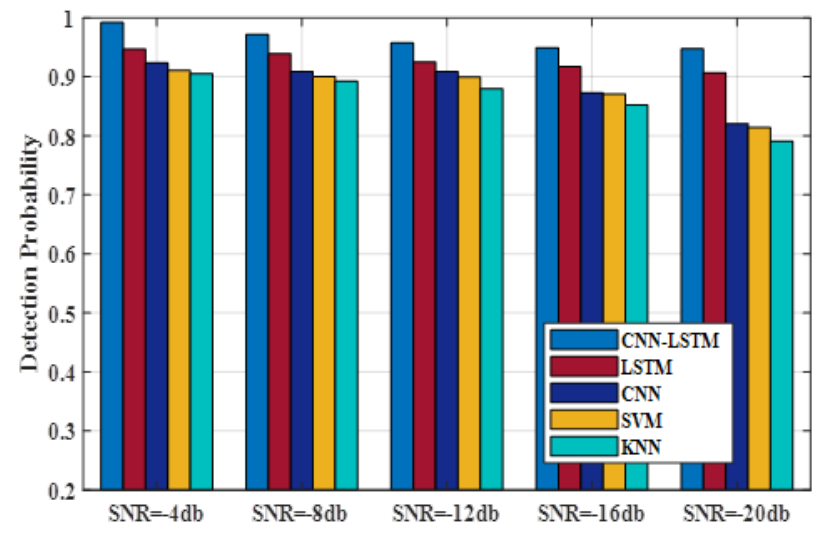

Fig. 10. Detection Probability

The effectiveness of the proposed CNN-LSTM algorithm is verified under various SNR ranges. A graph has been plotted between Pd (vs.) Signal to Noise Ratio for the five convolutional schemes and is shown in Figure.11. When compared to the existing methods namely CNN, LSTM, SVM and KNN, the proposed technique CNN LSTM has a high-performance detection algorithm. It is mainly caused due to the use of temporal dependency of the proposed technique where the existing methods fail to do so because CNN can predict only the spatial features. The supervised techniques like SVM and KNN have higher training module time. So, both the matrix correlation features are needed to find out the presence of primary user.

\section{Conclusion}

The cognitive radio system shows great impact in developing a good implementation of networks. The determinism includes both spectrum sensing and spectrum handoff algorithms. Though there are various spectrum sensing strategies demonstrated in the previous implementation, the technique which satisfies both ease of implementation and accuracy need to be developed. Hence, a spectrum sensing algorithm with improved CNN LSTM is proposed in this paper. The principle used in this approach is that any PU signal will have a modulation format associated with it and hence identifying the presence of a modulation format itself establishes the presence of PU signal. This approach is able to effectively sense the PUs presence. The experimental results are carried out in MATLAB. In this proposed technique, probability detection is considered as one of the important parameters for the efficiency of the method. Simulation results were carried out under different SNRs to indicate improved execution of the invented algorithm. The methodology can be additionally improved by using different optimizing techniques to determine the best hidden layer size of the network so that complexity can be further reduced thereby identifying PU presence more effectively. 


\section{References}

[1] H. M. A. Abdullah, and A. V. S. Kumar, "HFSA-SORA: Hybrid firefly simulated annealing based spectrum opportunistic routing algorithm for Cognitive Radio Ad hoc Networks (CRAHN)," in Proc. of 2017 International Conference on Intelligent Computing and Control (I2C2), 2017. Article (CrossRef Link)

[2] A. Agarwal, S. Dubey, M. A. Khan, R. Gangopadhyay, and S. Debnath, "Learning based primary user activity prediction in cognitive radio networks for efficient dynamic spectrum access," in Proc. of SPCOM, pp. 1-5, June 2016. Article (CrossRef Link)

[3] A. Al-Tahmeesschi, M. Lopez-Benitez, J. Lehtomaki, and K. Umebayashi, "Investigating the Estimation of Primary Occupancy Patterns under Imperfect Spectrum Sensing,” in Proc. of 2017 IEEE Wireless Communications and Networking Conference Workshops (WCNCW), 2017. Article (CrossRef Link)

[4] F. Azmat, Y. Chen, and N. Stocks, "Analysis of spectrum occupancy using machine learning algorithms," IEEE Trans. Veh. Technol, vol. 65, no. 9, pp. 6853-6860, September 2016.

Article (CrossRef Link)

[5] S. Badrinath, and V. U. Reddy, “A hybrid energy detection approach to spectrum sensing," in Proc. of 2009 First UK-India International Workshop on Cognitive Wireless Systems (UKIWCWS), December 2009. Article (CrossRef Link)

[6] Chun-Wei Tsai, Chien-Hui Hsia, Shuang-Jie Yang, Shih-Jui Liu and F. Zhi-Yan, "Optimizing hyperparameters of deep learning in predicting bus passengers based on simulated annealingang," Applied Soft Computing Journal, vol. 88, 2020. Article (CrossRef Link)

[7] C. Clancy, J. Hecker, E. Stuntebeck, and T. O'Shea, "Applications of machine learning to cognitive radio networks," IEEE Wireless Communication, vol. 14, no. 4, pp. 47-52, August 2007. Article (CrossRef Link)

[8] L. Deng, "A tutorial survey of architectures, algorithms, and applications for deep learning," APSIPA Trans. Signal Inform. Process, vol.3, pp.1-29, 2014. Article (CrossRef Link)

[9] Dhaval Patel and Yogesh Trivedi, "Non-parametric Blind Spectrum Sensing Based on Censored Observations for Cognitive radio,” Journal of Signal Processing Systems, vol. 78, pp. 275-281, March 2015. Article (CrossRef Link)

[10] A. Ghasemi, E. S. Sousa, "Collaborative spectrum sensing for opportunistic access in fading environments," in Proc. of the 1st IEEE International Symposium on New Frontiers in Dynamic Spectrum Access Networks (DySPAN '05), pp. 131-136, November 2005. Article (CrossRef Link)

[11] S. Hochreiter and J. Schmidhuber, "Long short-term memory," Neural Comput., vol. 9, no. 8, pp. 1735-1780, November 1997. Article (CrossRef Link)

[12] W. Huixin, M. Duo, and L. He, "Analysis and Simulation of the Dynamic Spectrum Allocation Based on Parallel Immune Optimization in Cognitive Wireless Networks,” The Scientific World Journal, pp. 1-8, 2014. Article (CrossRef Link)

[13] Hurmat Ali Shah and Insoo Koo, "Reliable Machine Learning Based Spectrum Sensing inCognitive Radio Networks,” Wireless Communications and Mobile Computing, 2018. Article (CrossRef Link)

[14] Jiandong Xie, Jun Fang, Chang Liu, Xuanheng Li, "Deep Learning-Based Spectrum Sensing in Cognitive Radio: A CNN-LSTM Approach," IEEE Communications Letters, vol. 24, no. 10, pp. 2196-2200, 2020. Article (CrossRef Link)

[15] Kai Yang, Zhitao Huang, Xiang Wang and Xueqiong Li, “A Blind Spectrum Sensing Method Based on Deep Learning,” Sensors, vol. 19, no. 10, pp. 2270, March 2019.

Article (CrossRef Link)

[16] Karaputugala Madushan Thilina, Kae Won Choi, Nazmus Saquib, and Ekram Hossain, "Machine Learning Techniques for Cooperative Spectrum Sensing in Cognitive Radio Networks," IEEE Journal on selected areas in communications, vol. 31, no. 11, pp. 2209-2221 November 2013. Article (CrossRef Link)

[17] S. Kirkpatrick, C.D. Gelatt, and M.P. Vecchi, “Optimization by simulated annealing,” Science, vol. 220, no. 4598, pp. 671-680, May 1983. Article (CrossRef Link) 
[18] A. Krizhevsky, I. Sutskever, and G. E. Hinton, "Imagenet classification with deep convolutional neural networks,” in Proc. of NIPS Stateline NV, pp. 1097-1105, December 2012.

[19] V. Kuppusamy, and R. Mahapatra, "Primary user detection in OFDM based MIMO Cognitive Radio," in Proc. of 3rd International Conference on Cognitive Radio Oriented Wireless Networks and Communications, 2008. Article (CrossRef Link)

[20] M. Lakshmi, R.Saravanan, and R.Muthaiah, "Energy Detection Based Spectrum Sensing For Cognitive Radio Using Fusion Rules,” International Journal of Scientific \& Engineering Research, vol. 4, no. 6, June 2013.

[21] M. Längkvist L. Karlsson, A. Loutfi, “A review of unsupervised feature learning and deep learning for time-series modelling,” Pattern Recognit, Lett., vol. 42, pp.11-24, 2014. Article (CrossRef Link)

[22] W. Lee, M. Kim, and D. H. Cho, “Deep Cooperative Sensing: Cooperative Spectrum Sensing Based on Convolutional Neural Networks,” IEEE Transactions on Vehicular Technology, vol. 68, no. 3, pp. 3005-3009, March 2019. Article (CrossRef Link)

[23] Z. C. Lipton, J. Berkowitz, and C. Elkan, "A critical review of recurrent neural networks for sequence learning," 2015.

[24] C. Liu, J. Wang, X. Liua, and Y. C. Liang, "Deep CM-CNN for Spectrum Sensing in Cognitive Radio,” IEEE Journal on Selected Areas in Communications, vol. 37, no. 10, pp. 2306-2321, October 2019.

[25] X. Lu, P. Wang, D. Niyato, and E. Hossain, "Dynamic Spectrum Access in Cognitive Radio Networks with RF Energy Harvesting,” IEEE Wirel. Communication, vol. 21, no. 3, pp. 102-110, 2014.

[26] Mangesh V Deshmukh, Dr. Mrs. Shruti Oza, "Spectrum Sensing based on Energy Detection for Cognitive Radio using FPGA,” vol. 08, no. 05, May 2019.

[27] Mingdong $\mathrm{Xu}$, Zhendong Yin, and Mingyang Wu, "Spectrum Sensing Based on Parallel CNN-LSTM Network," in Proc. of 2020 IEEE 91st Vehicular Technology Conference (VTC2020-Spring), 2020.

[28] J. Mitola and G. Q. Maguire, "Cognitive radio: Making software radios more personal," IEEE Pers. Commun., vol. 6, no. 4, pp. 13-18, August 1999. Article (CrossRef Link)

[29] Ouchra Senadji and Kevin Chang, "Detection of dynamic primary user with cooperative spectrum sensing,” in Proc. of 21st European Signal Processing Conference (EUSIPCO 2013), May 2014.

[30] Patel D. K. and Y. N. Trivedi, "LRS-G2G2 Based Non-parametric Spectrum Sensing for Cognitive Radio,” in Proc. of International Conference on Cognitive Radio Oriented Wireless Networks, vol. 172, pp 330-341, 2014.

[31] H, S. Reyes, N. Subramaniam, N. Kaabouch, and W. Chen, “A spectrum sensing technique based on autocorrelation and Euclidean distance and its comparison with energy detection for cognitive radio networks,” Comput. Electronics journal Eng., vol. 52, pp. 319-327, 2016. Article (CrossRef Link)

[32] Saber Mohammed, El Rharras Abdessamad, and Saadane Rachid, "An Optimized Spectrum Sensing Implementation based on SVM, KNN and TREE Algorithms,” in Proc. of International Conference on Signal-Image Technology \& Internet-Based Systems, 2019.

[33] P. Sharma and V. Abrol, "Individual vs cooperative spectrum sensing for Cognitive Radio Networks," in Proc. of 2013 Tenth International Conference on Wireless and Optical Communications Networks (WOCN), 2013.

[34] Shree Krishna Sharma, Mohammad Patwary, Symeon Chatzinotas, Bjorn Ottersten, and Mohamed Abdel-Maguid, "Repeater for 5G Wireless: A Complementary Contender for Spectrum Sensing Intelligence,”.

[35] S. Haykin, “Cognitive radio: brain-empowered wireless communications,” IEEE Journal on Selected Areas in Communications, vol. 23, no. 2, pp. 201-220, 2005. Article (CrossRef Link)

[36] K. Simonyan and A. Zisserman, "Very deep convolutional networks for large-scale image recognition,” arXiv preprint arXiv, pp.1409.1556, 2014. 
[37] Z. Sun, Q. Wang, and C. Che, "Study of Cognitive Radio Spectrum Detection in OFDM System," in Proc. of 2010 Asia-Pacific Conference on Wearable Computing Systems, 2010. Article (CrossRef Link)

[38] Y. J. Tang, Q. Y. Zhang, and W. Lin, "Artificial Neural Network Based Spectrum Sensing Method for Cognitive Radio," in Proc. of 2010 International Conference on Computational Intelligence and Software Engineering, 2010. Article (CrossRef Link)

[39] C. W. Tsai, C. H. Hsia, S. J. Yang, S. J. Liu, and Z. Y. Fang, "Optimizing hyper parameters of deep learning in predicting bus passengers based on simulated annealing," Applied Soft Computing Journal, vol. 88, no. 106068, 2020. Article (CrossRef Link)

[40] Vaibhav Kumar, Deep Chandra Kandpal, and Monika Jain, "K-mean Clustering based Cooperative SpectrumSensing in Generalized_- $\mu$ Fading Channels," in Proc. of 2016 Twenty Second National Conference on Communication (NCC), 2016. Article (CrossRef Link)

[41] H. Wang, E. H. Yang, Z. Zhao, and W. Zhang, "Spectrum sensing in cognitive radio using goodness of the testing," IEEE Trans.Wireless Communication, vol. 8, no. 11, pp. 5427-5430, November 2009.

[42] B. Wang and K. J. R. Liu, "Advances in cognitive radio networks: a survey,” IEEE Journal on Selected Topics in Signal Processing, vol. 5, no. 1, pp. 5-23, 2011. Article (CrossRef Link)

[43] Wangjam Niranjan Singh and Ningrinla Marchang, "A Review on Spectrum Allocation in Cognitive Radio Network," International Journal of Communication Networks and Distributed Systems, vol.23, no.2, pp.172 - 193, March 2018.

[44] J. B. Wei, S. Wang and H. T. Zhao, "Cognitive wireless networks: key techniques and sate of the art,” Journal on Communications, vol. 32, no. 11, pp. 147-158, 2011.

[45] Wei Li, Kai Liu, Lin Yan, Fei Cheng, YunQiu Lv, and LiZhe Zhang, "FRD-CNN: Object detection based on small-scale convolutional neural networks and feature reuse," Scientific Reports, vol. 9, no. 1, 2019. Article (CrossRef Link)

[46] Wei Ma, Mu Qing Wu, Dong Liu, and Meng Ling Wang, "User sensing based on MIMO cognitive radio sensor networks," in Proc. of 2009 2nd IEEE International Conference on Computer Science and Information Technology, 2009. Article (CrossRef Link)

[47] Xianghui Cao, Xiangwei Zhou, Lu Liu, and Yu Cheng, "Energy-Efficient Spectrum Sensing for Cognitive Radio Enabled Remote State Estimation Over Wireless Channels," IEEE Transactions on Wireless Communications, vol. 14, no. 4, April 2015.

[48] L. Xie and A. Yuille, "Genetic CNN," in Proc. of the IEEE International Conference on Computer Vision, pp. 1388-1397, 2017. Article (CrossRef Link)

[49] J. Xie, J. Fang, C. Liu, and X. Li, "Deep Learning-Based Spectrum Sensing in Cognitive Radio: A CNN-LSTM Approach,” IEEE Communications Letters, 2020.

[50] T. Xu, M. Zhang, and H. Hu, "Harmonious Coexistence of Heterogeneous Wireless Networks in Unlicensed Bands: Solutions from the Statistical Signal Transmission Technique," IEEE Vehicle. Tech. Mag, vol. 14, no. 2, pp. 61-69, June 2019. Article (CrossRef Link)

[51] R. G. Yelalwar, and Y. Ravinder, "Artificial Neural Network Based Approach for Spectrum Sensing in Cognitive Radio," in Proc. of International Conference on Wireless Communications, Signal Processing and Networking (WiSPNET), 2018. 

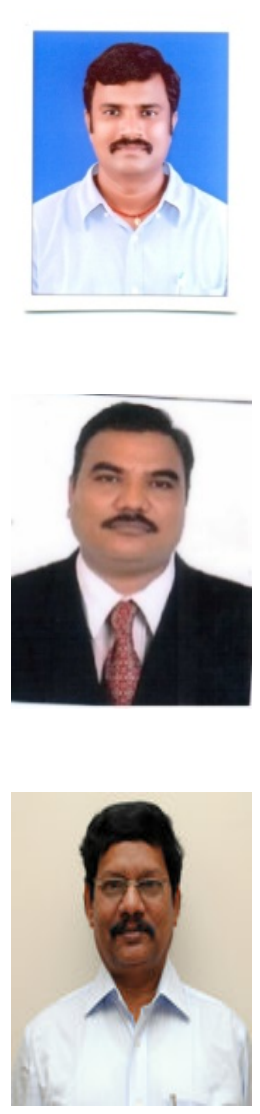

Appala Raju Uppala received the Diploma in Electronics and Communication Engineering from Govt. Polytechnic College, Narsipatnam in 1997, A.M.I.E Degree in ECE from Institution of Engineers India, Kolkata in 2004, and Master of Technology from Jawaharlal Nehru Technological University (JNTU), Hyderabad in 2007, and Pursuing the Ph.D degree from department of ECE, Jawaharlal Nehru Technological University (JNTU), Kakinada, India. He has published more than 12 technical papers in national and international journals and Conferences. His areas of interest Analog Electronics and Design, Cognitive Radio System, Communication and signal processing.

C. Venkata Narasimhulu received B.Tech degree in Electronics and Communication Engineering from S V University, Tirupathi in 1995 and Master of Technology in Instrumentation \& Control Systems from REC, Calicut in 2000 and Ph.D. from JNTU, Kakinada in 2013 in the area of signal Processing. He has published more than 45 technical papers in national and international Journals and Conferences. His interested areas are Signal Processing, Cognitive Radio, Image processing and medical image processing etc.

K. Satya Prasad received B Tech. degree in Electronics and Communication Engineering from JNTU college of Engineering, Anantapur, in 1977 and M. E. degree in Communication Systems from Guindy college of Engineering, Madras University, in 1979 and Ph.D from Indian Institute of Technology, Madras in 1989. He has published more than 150 technical papers in different National \& International conferences and Journals and Authored one Text book. His areas of Research include Communications Signal Processing, Image Processing, Speech Processing, Neural Networks \& Ad-hoc wireless networks etc. 